\title{
Laser-induced control of an electronic nematic quantum phase transition
}

\author{
Avraham Klein $\odot$, Morten H. Christensen $\odot$, and Rafael M. Fernandes $\odot$ \\ School of Physics and Astronomy, University of Minnesota, Minneapolis, Minnesota 55455, USA
}

(Received 4 October 2019; revised manuscript received 9 February 2020; accepted 12 February 2020; published 18 March 2020; corrected 15 July 2020)

\begin{abstract}
Ultrafast techniques have emerged as promising methods to study and control quantum materials. To maintain the quantum nature of the systems under study, excess heating must be avoided. In this paper, we demonstrate a method that employs the nonequilibrium laser excitation of planar-stretching optical phonons in tetragonal systems to quench an electronic nematic state across a quantum phase transition. Appropriately tuned offresonant pulses can perform a quantum quench of the system either into the nematic phase (red detuning) or out of it (blue detuning). The nonlinear coupling of this phonon mode to nematicity not only mediates interactions in the nematic channel, but it also suppresses heating effects. We illustrate the applicability of our general results by considering the microscopic parameters of the nematic unconventional superconductor FeSe.
\end{abstract}

DOI: 10.1103/PhysRevResearch.2.013336

\section{INTRODUCTION}

Nonequilibrium studies of strongly correlated electron systems have undergone significant progress in recent years, due to both theoretical advances [1-3] and outstanding developments in ultrafast pump-probe techniques [4-7]. Light-based control of quantum materials has wide applications ranging from high-temperature superconductivity [6-8] to quantum computation [9]. The basis for such control derives from the use of laser pulses to switch between different electronic states inhabiting the phase diagrams of these exotic materials. A natural route to realizing such control is to exploit the electron-phonon coupling and precisely excite infraredactive optical phonon modes [5,10-13] via strong terahertz laser pulses [14-17]. Because infrared phonon modes do not couple directly to the electronic charge density, the changes in electronic properties are mediated by nonlinear effects [18-20]. Importantly, the ordered states that can be most efficiently controlled by this approach are those that couple strongly to the lattice, such as superconductivity or metalto-insulator transitions [8,19-21]. Identifying a method to optically control a strongly correlated electronic phase, while maintaining its quantum, low temperature properties, would provide a key ingredient for manipulating the intertwined orders characteristic of quantum materials.

Electronic nematicity is intimately coupled to the lattice [22-24]. When the electronic system spontaneously breaks rotational symmetry [25], it inevitably triggers a structural distortion. Nematic phases have been widely observed in quantum materials, from unconventional superconductors such as cuprates, pnictides, and heavy fermions, to ruthenates and

Published by the American Physical Society under the terms of the Creative Commons Attribution 4.0 International license. Further distribution of this work must maintain attribution to the author(s) and the published article's title, journal citation, and DOI. semiconductors displaying the quantum Hall effect [26-29]. The driving mechanism of nematicity in these compounds and its relationship with other phenomena such as superconductivity, magnetism, and charge-order, are all hotly debated [29-31]. Most suggestively, in several of these materials it is widely believed that the nematic phase ends at a putative quantum critical point (QCP) [32], which may host an exotic non-Fermi-liquid phase and a superconducting dome [33]. Controlling the nematic degrees of freedom would therefore provide a high-precision tool for studying the competing and intertwined orders in these systems. The strong coupling to the lattice [34] suggests light control as a feasible route, which has hitherto remained largely unexplored.

In this paper, we develop a theory of nonequilibrium optical control of a generic nematic phase on the square lattice, based on the off-resonance excitation of a particular infrared optical phonon mode. Being entirely based on symmetry arguments, our model is independent of microscopic considerations, and is thus applicable to a wide variety of nematic materials. In particular, we demonstrate how our results offer an avenue to implement a laser-controlled quench across the nematic quantum phase transition, as illustrated schematically in Fig. 1. This can be used to either promote nematic order in an otherwise disordered state or to suppress nematic order to enhance other competing states, such as superconductivity [35]. We illustrate the applicability of our model by considering microscopic parameters of the unconventional superconductor FeSe, a poster child of electronic nematic order.

The key ingredient in our analysis is that the onset of nematic order breaks the tetragonal symmetry either by making the horizontal and vertical bonds inequivalent (called $d_{x^{2}-y^{2}}$ wave, or $B_{1 g}$, nematic order) or the diagonals inequivalent (called $d_{x y}$-wave, or $B_{2 g}$, nematic order). For concreteness, we consider hereafter the $B_{1 g}$ case. The orthorhombic lattice distortion that accompanies nematic order is due to the linear coupling between the electrons and a transverse acoustic phonon mode that propagates along the [110] direction. The phonon velocity is strongly renormalized by nematic 
(a)

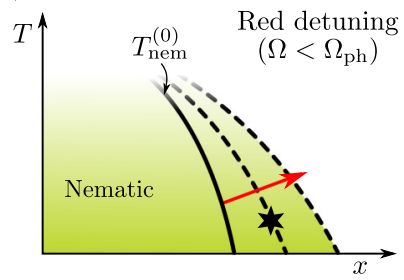

(b)

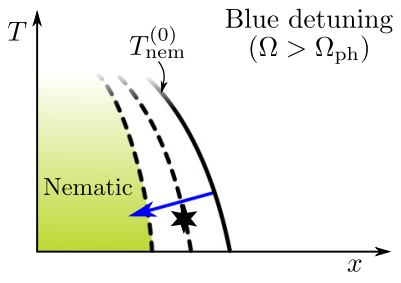

FIG. 1. Schematic illustration of the proposed experimental setup. Depending on the detuning between the applied optical pulse and the $E_{u}$ phonon frequency, $\Omega_{\mathrm{ph}}$, the nematic phase can be either enhanced [(a), red detuning] or suppressed [(b), blue detuning]. The solid line denotes the equilibrium nematic phase transition at $T_{\mathrm{nem}}^{(0)}$, while the dashed lines illustrate the nonequilibrium shift as the laser intensity is increased. The stars denote compositions that would undergo a nonequilibrium nematic transition.

fluctuations and vanishes at the nematic transition. Although this linear nematoelastic coupling has been widely employed to investigate the nematic properties of many materials [28,32], the acoustic nature of the phonon makes it inconvenient for optical control [36]. Instead, we propose to control the nematic phase by optically exciting the $E_{u}$ optical phonon mode, corresponding to two degenerate planar stretching lattice vibrations. This infrared-active mode, ideal for laser manipulation, is present in any tetragonal system. While symmetry forbids incoming photons and the $E_{u}$ phonon from coupling linearly to the electronic density, the $E_{u}$ vibrations do couple quadratically to the electronic nematic degrees of freedom. Therefore, in contrast to traditional pump-probe setups, the lattice is excited directly, whereas the electronic nematic channel is only excited indirectly, via the nonlinear phonon coupling. As a result, one expects heating effects, which commonly plague the interpretation of pump-probe data, to be significantly reduced. Our proposal is thus similar in spirit to that of Ref. [20], which proposed that the excitation of infrared-active phonons leads to an attractive electronic interaction that favors superconductivity via the nonlinear coupling to the charge density.

The differences between a quadratic versus a linear coupling also play a crucial role in determining how a nematic phase transition can be controlled by externally applied lattice perturbations. Physically, the linear coupling between electrons and the acoustic phonon is manifested by a change in the rest positions of the atoms of the square lattice once nematic order onsets. As a result, lattice displacements induced by external strain act as a conjugate field to the nematic order parameter. Thus, although strain can be used to assess the nematic susceptibility, it smears the nematic transition completely, making it difficult to manipulate a nematic transition with strain. In contrast, the quadratic coupling to the optical phonon is manifested as a splitting of the resonance frequencies of the degenerate $E_{u}$ phonon modes $[20,37]$ in the presence of nematic order. Consequently, the external excitation of this phonon mode shifts the frequency of the lowenergy collective nematic mode, without breaking explicitly the tetragonal symmetry of the system.

As we will show, the amplitude and phase of this shift can be controlled in a precise manner by an external optical

pulse and thus used to tune the system both toward and away from the nematic transition, in proportion to the intensity of the exciting laser. The experimental consequences are shown in Fig. 1. For laser frequencies below the phonon resonance frequency, $\Omega<\Omega_{\mathrm{ph}}$, the nematic phase is enhanced, while for $\Omega>\Omega_{\mathrm{ph}}$ it is suppressed.

To derive these results, we start from a general model for coupled nematic degrees of freedom and $E_{u}$ phonons. We are not concerned with the microscopic mechanism of the nematic transition, and merely describe it by a bosonic field $\phi$ that transforms as the $B_{1 g}$ irreducible representation of the tetragonal point group. In terms of the electronic field operators $\psi(\mathbf{k}, v)$, the nematic bosonic field is proportional to the quadrupolar charge density, given by $\phi(\mathbf{q}, \omega) \sim \sum_{\mathbf{k}, v}\left(k_{x}^{2}-\right.$ $\left.k_{y}^{2}\right) \psi^{\dagger}(\mathbf{k}+\mathbf{q} / 2, v+\omega / 2) \psi(\mathbf{k}-\mathbf{q} / 2, v-\omega / 2)$.

We describe the phonons by an optical degenerate mode $\mathbf{X}=\left(X_{1}, X_{2}\right)$ which couples to an electric field $\boldsymbol{\epsilon}=\left(\varepsilon_{1}, \varepsilon_{2}\right)$. For concreteness, we assume our system is on a twodimensional lattice, and is described by the coupled action,

$$
\hbar \mathcal{S}=\int_{\mathcal{C}} d t \sum_{\mathbf{x}_{i}}\left[\mathcal{L}_{\mathrm{ph}}+\mathcal{L}_{\mathrm{nem}}+\mathcal{L}_{\mathrm{int}}\right]
$$

where $\mathcal{C}$ is the Keldysh contour, and

$$
\begin{gathered}
\mathcal{L}_{\mathrm{nem}}=\phi \frac{1}{2 \chi_{0}}\left(-\partial_{t}^{2}-r\right) \phi-\frac{u}{4 \chi_{0}} \phi^{4}, \\
\mathcal{L}_{\mathrm{ph}}=\frac{M}{2} \mathbf{X}\left(-\partial_{t}^{2}-\Omega_{\mathrm{ph}}^{2}\right) \cdot \mathbf{X}+q \mathbf{X} \cdot \boldsymbol{\epsilon}, \\
\mathcal{L}_{\text {int }}=-\frac{\lambda}{a^{2}} \phi(x)\left(X_{1}^{2}-X_{2}^{2}\right) .
\end{gathered}
$$

Here, $r$ and $u$ have units of frequency squared and describe a Landau theory for the nematic critical point that occurs at $r=0$. Thus, $\sqrt{r}$ should be understood as the frequency of the nematic collective mode, which softens at the nematic transition. For a derivation of this action in the context of FeSe see, e.g., Refs. [22,29,38]. $M, \Omega_{\mathrm{ph}}$, and $q$ describe the mass, resonance frequency, and ionic charge of the $E_{u}$ mode, which we approximate as an Einstein phonon due to its optical nature. $\lambda$ is the effective coupling and $a=\sqrt{\hbar / M \Omega_{\mathrm{ph}}}$ is the phonon oscillator length. We choose $\chi_{0}$ such that $\phi$ is dimensionless.

Our main result is that due to the electric field excitation, the frequency of the nematic mode is modified. For the simplest case of a system right above the nematic transition, with a monochromatic laser field that does not explicitly break the symmetry of the lattice (e.g., a linearly $45^{\circ}$ polarized beam), the modification takes the form

$$
r \rightarrow r+r_{\varepsilon}
$$

where

$$
r_{\varepsilon} \propto|\boldsymbol{\epsilon}|^{2}\left(\Omega^{2} / \Omega_{\mathrm{ph}}^{2}-1\right) .
$$

In other words, the nematic frequency is softened or hardened in proportion to the beam intensity and the detuning from the photon resonance, pushing the system either to or away from an instability. As we will show, the result of this modification is that at a critical field strength $|\boldsymbol{\epsilon}|=\varepsilon_{c}$ of a red-detuned beam, a system in the tetragonal phase becomes unstable to 
nematic order. Similarly, a blue detuned beam has a critical strength for which the static order parameter of a system in the nematic phase become zero. In general, the behavior of the nematic field in response to the laser excitation is more complicated than the specific result in Eq. (5), but the overall behavior is the same: There is a critical field strength for which a red-detuned beam pushes the system into a nematic phase and a critical strength for which a blue-detuned beam pushes the system out of a nematic phase.

The rest of the paper is organized as follows. In Sec. II, we analyze the action of Eqs. (1)-(4) and derive the modification of the nematic response for a monochromatic beam. In Sec. III, we generalize our results to a laser pulse of a finite width and study the full effective action for the nematic mode. In Sec. IV, we describe how to apply our results to the Fe-based superconductor FeSe.

\section{DERIVATION OF THE EFFECTIVE NEMATIC RESPONSE}

\section{A. The nematic effective action}

We start with the model introduced in the introduction, Eq. (1). The model is defined on the Keldysh contour to account for the nonequilibrium effects of the electric field. In the usual manner [39], we split $\mathcal{C}$ into backward $(<)$ and forward $(>)$ segments, and perform the Keldysh rotation for the fields, i.e., $\phi^{\mathrm{cl}, \mathrm{q}}=\left(\phi_{>} \pm \phi_{<}\right) / 2$. Then we integrate out the phonon modes and obtain an effective action for the nematic mode,

$$
S_{\text {nem }}=\sum_{\mathbf{x}_{i}} \operatorname{Tr}\left[\frac{1}{2} \Phi \chi_{\text {nem }}^{-1} \Phi-\frac{q^{2}}{2} \mathcal{E} \tau^{1} D \tau^{1} \mathcal{E}\right],
$$

where the trace is over (Euclidean) time and

$$
D=\left[D_{0}^{-1}-2 \frac{\lambda}{a^{2}} \tilde{\Phi} \sigma^{z}\right]^{-1}-D_{0} .
$$

Here, $\Phi=\left(\phi^{\mathrm{cl}}, \phi^{\mathrm{q}}\right), \tilde{\Phi}=\tau^{1} \phi^{\mathrm{cl}}+\tau^{0} \phi^{\mathrm{q}}, \mathcal{E}=\left(\boldsymbol{\epsilon}^{\mathrm{cl}}, \boldsymbol{\epsilon}^{\mathrm{q}}\right), \boldsymbol{\tau}$, and $\sigma$ are Pauli matrices in Keldysh and coordinate space, respectively, and $D_{0}$ and $\chi_{\text {nem }}$ are the equilibrium propagators of the phonon and nematic modes, whose retarded components are given by the $(\mathrm{cl}, \mathrm{q})$ component of the matrix propagators,

$$
\begin{gathered}
D^{R}(\Omega) \equiv D_{0}^{(\mathrm{cl}, \mathrm{q})}(\Omega)=\frac{M^{-1}}{\Omega^{2}-\Omega_{\mathrm{ph}}^{2}+i \Gamma_{\mathrm{ph}} \Omega} \sigma^{0}, \\
\chi^{R}(\omega)=\chi_{0}\left(\omega^{2}-r+i \Gamma_{\mathrm{nem}} \omega\right)^{-1} .
\end{gathered}
$$

$\Gamma_{\text {nem }}$ and $\Gamma_{\mathrm{ph}}$ are phenomenological damping terms, arising from, e.g., Landau damping. Explicitly, $D_{0}$ has the form

$$
D_{0}=\left(\begin{array}{cc}
D^{K} & D^{R} \\
D^{A} & 0
\end{array}\right) \otimes \sigma^{0}
$$

Equation (7) is our first main result. It demonstrates that the phonons modify the nematic action in proportion to the laser intensity $|\mathcal{E}|^{2}$. To proceed further analytically, we expand $D$ perturbatively to second order in $\phi$. The expansion is justified for $|\phi| \ll 1$, and henceforth we always assume we are in this regime (the expansion is also justified, even for $|\phi| \sim 1$, when $\lambda / \Omega_{\mathrm{ph}} \ll 1$ and the field frequencies are not too close to the phonon resonance). The leading contributions to the action are

$$
\begin{gathered}
\delta S^{(1)}=-\frac{\lambda q^{2}}{a^{2}} \operatorname{Tr} \mathcal{E} \tau^{1} D_{0} \tilde{\Phi} D_{0} \sigma^{z} \tau^{1} \mathcal{E}, \\
\delta S^{(2)}=-\frac{2 \lambda^{2} q^{2}}{a^{4}} \operatorname{Tr} \mathcal{E} \tau^{1} D_{0} \tilde{\Phi} D_{0} \tilde{\Phi} D_{0} \tau^{1} \mathcal{E} .
\end{gathered}
$$

Equation (12) implies that the linear term in $\Phi$ vanishes when the laser excites both $E_{u}$ modes equally (e.g., for circularly polarized or $45^{\circ}$ polarized light). This is a consequence of the trace over $\sigma^{z}$, which yields a term proportional to $\varepsilon_{1}-\varepsilon_{2}$. Then the only contribution is the quadratic term, Eq. (13), which corresponds to a shift $\delta \chi^{-1}$ to the inverse nematic susceptibility $\chi_{\text {nem }}^{-1}$ in Eq. (7).

While the results above are general, we now focus on the physically interesting case of a nematic mode that is much slower than the phonons $r \ll \Omega_{\mathrm{ph}}^{2}$, as expected near a nematic phase transition, and assume a classical laser field $\epsilon^{q} \approx 0$. For definiteness, we choose a $45^{\circ}$ polarized beam with $\epsilon_{1}=\epsilon_{2}=$ $\varepsilon^{\mathrm{cl}}(t)$. Explicitly, Eq. (13) has the form

$$
\begin{aligned}
& \delta S^{(2)}=-\frac{2 q^{2} \lambda^{4}}{a^{4}} \int \frac{d \omega_{1} d \omega_{2} d \nu}{(2 \pi)^{3}} \varepsilon^{\mathrm{cl}}\left(\omega_{1}-v\right) D^{A}\left(v-\omega_{1}\right) D^{R}\left(v-\omega_{2}\right) \varepsilon^{\mathrm{cl}}\left(v-\omega_{2}\right) \\
& \times\left(\phi^{\mathrm{cl}}\left(-\omega_{1}\right) \quad \phi^{q}\left(-\omega_{1}\right)\right) \cdot\left(\begin{array}{cc}
0 & D^{A}(v) \\
D^{R}(v) & D^{K}(v)
\end{array}\right) \cdot\left(\begin{array}{l}
\phi^{\mathrm{cl}}\left(\omega_{2}\right) \\
\phi^{q}\left(\omega_{2}\right)
\end{array}\right) .
\end{aligned}
$$

Equation (14) gives a correction to the inverse nematic susceptibility $\delta \chi^{-1}$, which can be computed for any pulse shape. We discuss it in more detail later in this section. For now, we specialize to the case of a monochromatic beam:

$$
\varepsilon^{\mathrm{cl}}(t)=\frac{1}{\sqrt{2}} \varepsilon_{0} \cos \Omega t
$$

Plugging the Fourier transform of Eq. (15) into Eq. (14), we find the particular action term from which we can read out the retarded nematic susceptibility:

$$
\begin{aligned}
\delta \tilde{S}^{(2)}= & -\frac{q^{2} \lambda^{2} \varepsilon_{0}^{2}}{2 a^{4}}\left|D^{R}(\Omega)\right|^{2} \int \frac{d \omega}{2 \pi} \phi^{q}(-\omega)\left[D^{R}(\omega+\Omega)\right. \\
& \left.+D^{R}(\omega-\Omega)\right] \phi^{\mathrm{cl}}(\omega) .
\end{aligned}
$$

Here we have neglected terms with a large frequency transfer, of order $2 \Omega_{\mathrm{ph}}$. Neglecting these terms is justified if the relevant frequencies of the nematic system, i.e., $\sqrt{r}, \Gamma_{\text {nem }}$ are smaller than $\Omega_{\mathrm{ph}}$. 


\section{B. The collective modes of the nematic field}

The integrand of Eq. (16) is nothing but the induced correction to the inverse retarded nematic susceptibility $\left(\delta \chi^{-1}\right)^{R}(\omega)$, which is added to the bare retarded susceptibility in Eq. (10). The poles of the renormalized retarded nematic susceptibility $\left(\tilde{\chi}^{-1}\right)^{R}=\left(\chi^{-1}\right)^{R}+\left(\delta \chi^{-1}\right)^{R}$ are then given by

$$
\omega^{2}=r-i \Gamma_{\mathrm{nem}} \omega+\frac{\lambda^{2} \chi_{0}}{\hbar \Omega_{\mathrm{ph}}} n_{\mathrm{ph}}\left[d^{R}(\omega+\Omega)+d^{R}(\omega-\Omega)\right],
$$

where $n_{\mathrm{ph}}=\left\langle X_{1}^{2}+X_{2}^{2}\right\rangle / a^{2} \approx\left|D^{R}(\Omega)\right|^{2} q^{2} \varepsilon_{0}^{2} / a^{2}$ is the average phonon occupation number and $d^{R}=M \Omega_{\mathrm{ph}}^{2} D^{R}$ is a normalized propagator. Equation (17) has six solutions. To gain insight into them, we consider the classical equations of motion for the coupled phonon-nematic system:

$$
\begin{gathered}
\ddot{X}_{i}+\Gamma_{\mathrm{ph}} \dot{X}_{i}+\left(\Omega_{\mathrm{ph}}^{2} \pm \frac{2 \lambda}{M a^{2}} \phi\right) X_{i}=\frac{q \varepsilon_{0}}{\sqrt{2} M} \cos \Omega t, \\
\ddot{\phi}+\Gamma_{\mathrm{nem}} \dot{\phi}+r \phi+u \phi^{3}+\frac{\lambda \chi_{0}}{a^{2}}\left(X_{1}^{2}-X_{2}^{2}\right)=0 .
\end{gathered}
$$

Substituting the ansatz $\phi(t)=\operatorname{Re} \phi_{0} e^{-i \omega t}$, the values of $\omega$ that solve the classical equations coincide with the poles of Eq. (17). Equation (19) is very similar to the three-wave mixing problem, typical of cavity quantum-electrodynamic applications [40]. What is special in our case is that the three-wave mixing term is proportional to $X_{1}^{2}-X_{2}^{2}$, so it vanishes unless the nonlinear feedback from $\phi$ is accounted for. The first set of poles corresponds to a correction of the "bare" frequency $\omega=\sqrt{r}$ of the nematic collective mode. If $\sqrt{r} \ll\left\{\Omega_{\mathrm{ph}}, \Gamma_{\mathrm{ph}}, \Omega\right\}$ is the smallest scale in the problem, we may expand the propagators in Eq. (17) in $\omega$ and find

$$
-\omega^{2}-i\left(\Gamma_{\mathrm{nem}}+\Gamma_{\varepsilon}\right) \omega+\left(r+r_{\varepsilon}\right) \approx 0,
$$

where

$$
\begin{gathered}
r_{\varepsilon}=-n_{\mathrm{ph}} \frac{2 \lambda^{2} \chi_{0}}{\hbar \Omega_{\mathrm{ph}}}\left|d^{R}(\Omega)\right|^{2}\left(1-\Omega^{2} / \Omega_{\mathrm{ph}}^{2}\right), \\
\Gamma_{\varepsilon}=\Gamma_{\mathrm{ph}} n_{\mathrm{ph}} \frac{2 \lambda^{2} \chi_{0}}{\hbar \Omega_{\mathrm{ph}}^{3}}\left|d^{R}(\Omega)\right|^{4} \frac{2\left(\Omega_{\mathrm{ph}}^{4}-\Omega^{4}\right)-\left(\Omega_{\mathrm{ph}}^{2}-\Omega^{2}\right)^{2}}{\Omega_{\mathrm{ph}}^{4}} .
\end{gathered}
$$

This is our second main result, showing that $r$ is shifted either to or away from the nematic transition depending on the detuning between the laser frequency $\Omega$ and the phonon frequency $\Omega_{\mathrm{ph}}$, and in proportion to the pulse intensity $\varepsilon_{0}^{2}$.

Figure 2 depicts the evolution of these poles for a slightly red-detuned pulse. As $\varepsilon_{0}$ increases, the nematic mode frequencies soften toward zero and eventually split and become imaginary. At a critical value $\varepsilon_{0}=\varepsilon_{c}$, one of these imaginary poles crosses the real axis, signaling an instability toward nematic order, as shown by the time evolution of the classical solution $\phi(t)=\phi_{0} e^{-i \omega t}$ depicted in the inset.

The figure also displays other poles of $\tilde{\chi}^{R}$ from Eq. (17). In addition to the pair of poles discussed above, there are four additional poles corresponding approximately to the poles of the phonon propagators $D^{R}(\Omega+\omega)$ and $D^{R}(\omega-\Omega)$, which are therefore purely dynamic and nonperturbative in nature. For small detuning $\delta=\Omega_{\mathrm{ph}}-\Omega \ll \Omega$, two of the poles are

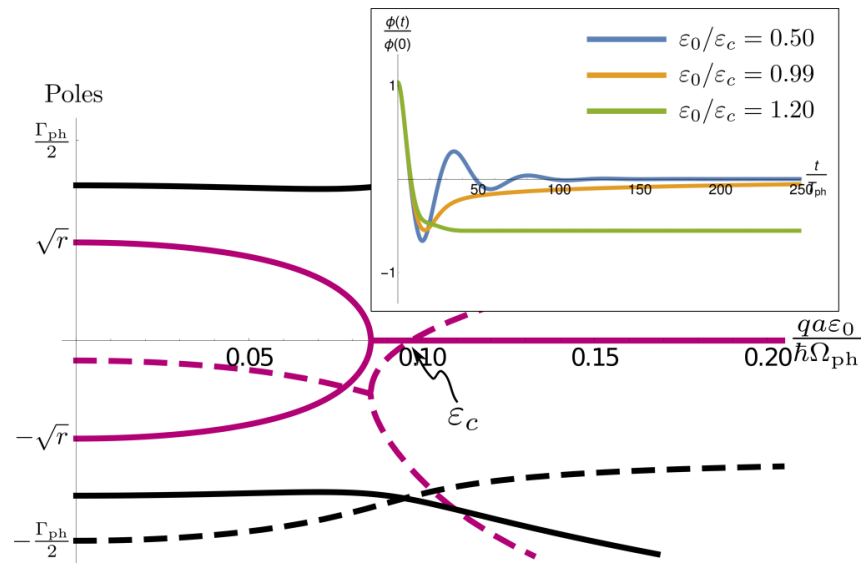

FIG. 2. Evolution of the low-lying poles of the nematic susceptibility as function of the intensity of the applied electric field $\varepsilon_{0}$. Solid (dashed) lines denote the real (imaginary) parts of the poles. The magenta trace is a pole that is softening due to the applied field, triggering an instability when its imaginary part becomes positive at the critical field strength $\varepsilon_{c}$. The inset depicts the classical evolution of $\phi(t)$ for several $\varepsilon_{0}$ values. The parameters used are given in Appendix A.

of order $\pm 2 \Omega_{\mathrm{ph}}$ and do not contribute to the slow dynamics of the nematic mode. The other poles obey $\omega \approx-i \frac{\Gamma_{\mathrm{ph}}}{2} \pm$ $\delta \sqrt{1-n_{p h} \frac{\lambda^{2} \chi_{0}}{\hbar r \delta}}$. Note that the residue of these four additional poles vanish as $\varepsilon_{0} \rightarrow 0$.

The shifting of the nematic mode $r_{\varepsilon}$ in Eq. (21) has a simple physical interpretation. The situation when $\sqrt{r}$ is the smallest frequency in the system corresponds to a type of inverse Born-Oppenheimer problem, where the electronic dynamics is much slower than the ionic (phononic) dynamics. The rapid phonon oscillations can then be integrated out, leaving a quasistatic contribution to the effective nematic excitation energy. As long as the lattice symmetry is not explicitly broken by these oscillations-i.e., the first-order contribution in Eq. (12) vanishes - the net effect is a slowdown or speedup of the nematic fluctuations.

The evolution of the poles and nematic order parameter can be found also for many other cases, such as when $\sqrt{r}$ is not the smallest frequency in the system. A particular case of interest is when $\Gamma_{\text {nem }} \gg \sqrt{r}$, i.e., the nematic mode is overdamped. This is the case in the Fe-based superconductors. The analysis is slightly modified from our discussion above, but the qualitative behavior is the same. We present this and a variety of other cases in Appendix A. For now, we finish this section by studying the behavior of a system with $r<0$, i.e., in the nematic phase, for a blue detuned beam.

In the ordered phase, the steady-state value of the nematic mode at zero field is nonzero, making the solutions of Eqs. (18) and (19) more complex. However, as long as $\phi$ is small, we can linearize the equations and obtain analytic solutions. For concreteness, consider the case where $\sqrt{|r|}$ is the smallest scale in the problem, i.e., we are approaching the nematic transition from the ordered side. Then the equation of motion for $\phi$ becomes

$$
\ddot{\phi}+\left(\Gamma_{\mathrm{nem}}+\Gamma_{\varepsilon}\right) \dot{\phi}+\left(r+r_{\varepsilon}\right) \phi+u \phi^{3}=0,
$$




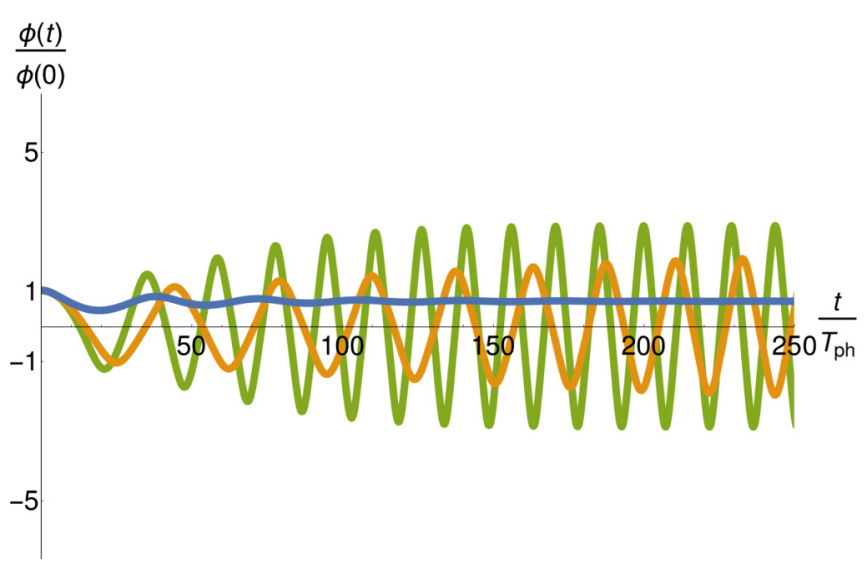

FIG. 3. Classical evolution of the nematic order parameter for the case where $r<0$, and the laser is used to quench the nematic order. The parameters used are given in Appendix A. The color code is the same as in Fig. 2.

where $\Gamma_{\varepsilon}, r_{\varepsilon}$ were defined in Eqs. (21) and (22). The static order parameter takes the value

$$
\langle\phi\rangle= \pm \sqrt{\frac{-\left(r+r_{\varepsilon}\right)}{u}},
$$

where the sign is determined by initial conditions. Linearizing around the static order parameter value, $\phi(t)=\langle\phi\rangle+\varphi$, we find

$$
\ddot{\varphi}+\left(\Gamma_{\text {nem }}+\Gamma_{\varepsilon}\right) \dot{\varphi}-2\left(r+r_{\varepsilon}\right) \varphi=0,
$$

which describes stable oscillations around the order parameter value. Figure 3, the evolution of $\varphi$ for some characteristic parameters, shows how the nematic order goes away with increased field.

\section{THE FULL NEMATIC EFFECTIVE ACTION}

In the previous section, we studied the shift of the poles of the nematic susceptibility for a monochromatic beam. In actual experiments, the laser is pulsed, i.e., $\varepsilon(t)=$ $\varepsilon_{0} \cos (\Omega t) e^{-|t| / \tau_{\text {pulse }}}$, where $\tau_{\text {pulse }}$ controls the duration of the pulse. To properly account for the behavior in the physical situation, we must study the full induced effective action in Eq. (14).

We start by explicitly writing out the effective action as

$$
\begin{aligned}
S^{(2)}= & \int \frac{d \omega_{1} d \omega_{2}}{2 \pi} \Phi\left(-\omega_{1}\right)\left[\chi_{\mathrm{nem}}^{-1}\left(\omega_{1}\right) \delta\left(\omega_{1}-\omega_{2}\right)\right. \\
& \left.+\delta \chi^{-1}\left(\omega_{1}, \omega_{2}\right)\right] \Phi\left(\omega_{2}\right),
\end{aligned}
$$

where we recall that

$$
\chi_{\mathrm{nem}}(\omega)=\left(\begin{array}{cc}
\chi^{K}(\omega) & \chi^{R}(\omega) \\
\chi^{A}(\omega) & 0
\end{array}\right), \quad \Phi(\omega)=\left(\begin{array}{c}
\phi^{\mathrm{cl}} \\
\phi^{\mathrm{q}}
\end{array}\right),
$$

and the correction to the inverse nematic susceptibility, as shown in Eq. (16), is

$$
\begin{aligned}
\delta \chi^{-1}\left(\omega_{1}, \omega_{2}\right)= & -\frac{2 q^{2} \lambda^{4}}{a^{4}} \int \frac{d v}{(2 \pi)^{2}} \varepsilon^{\mathrm{cl}}\left(\omega_{1}-v\right) D^{A}\left(v-\omega_{1}\right) \\
& \times\left(\begin{array}{cc}
0 & D^{A}(v) \\
D^{R}(v) & D^{K}(v)
\end{array}\right) D^{R}\left(v-\omega_{2}\right) \varepsilon^{\mathrm{cl}}\left(v-\omega_{2}\right) .
\end{aligned}
$$

To understand the meaning of Eq. (28), it is convenient to consider the situation when $\delta \chi^{-1}\left(\omega_{1}, \omega_{2}\right)=F\left(\omega_{1}\right) \delta\left(\omega_{1}-\omega_{2}\right)$ is a function of just one frequency. It can be verified that this is the case when the laser beam has only one component, $\epsilon^{\mathrm{cl}}(t) \propto e^{-i \Omega t}$, such that the integrations in Eq. (28) are constrained to $\omega_{1}=\omega_{2}, v-\omega_{2}=\Omega$. In that case the action, Eq. (26), depends only on one parameter, and furthermore obeys the fluctuation-dissipation theorem (FDT) at equilibrium [39],

$$
\tilde{\chi}^{K}(\omega)=\sigma(\omega)\left(\tilde{\chi}^{R}(\omega)-\tilde{\chi}^{A}(\omega)\right),
$$

where $\tilde{\chi}=\left(\chi^{-1}+\delta \chi^{-1}\right)^{-1}$ is the renormalized nematic susceptibility and $\sigma(\omega)$ is the sign function, which is the zerotemperature limit of the Bose-Einstein distribution function. Equation (29) follows from the fact that both $\chi$ and $D$ obey the equilibrium FDT separately. The implication is that a pure monochromatic laser induces a quasiequilibrium action for the nematic mode, provided that the nematic mode is irrelevant at frequencies of order $2 \Omega$ and that superposition between positive and negative frequency elements can be neglected, as in, e.g., a rotating-wave approximation. Recall that the electric field, being real, always has at least two frequencies, $\pm \Omega$.

To study the effect of a light pulse which has a finite envelope, it is convenient to transform to the frequency difference and $\operatorname{sum} \omega^{\prime}=\omega_{1}-\omega_{2}, \omega=\left(\omega_{1}+\omega_{2}\right) / 2$, so

$$
\begin{aligned}
\delta \chi^{-1}\left(\omega, \omega^{\prime}\right)= & -\frac{2 q^{2} \lambda^{4}}{a^{4}} \int \frac{d v}{(2 \pi)^{2}} \varepsilon^{c l}\left(\omega+\omega^{\prime} / 2-v\right) \\
& \times D^{A}\left(v-\omega-\omega^{\prime} / 2\right)\left(\begin{array}{cc}
0 & D^{A}(v) \\
D^{R}(v) & D^{K}(v)
\end{array}\right) \\
& \times D^{R}\left(v-\omega+\omega^{\prime} / 2\right) \varepsilon^{c l}\left(v-\omega+\omega^{\prime} / 2\right) .
\end{aligned}
$$

If $\delta \chi^{-1} \propto \delta\left(\omega^{\prime}\right)$, the system is in quasiequilibrium. For a slow enough pulse of length $\tau_{\text {pulse }}$ much greater than the inherent timescales of the problem, dictated by $\Gamma_{\mathrm{ph}}, \Gamma_{\mathrm{nem}}$, etc., the $\omega^{\prime}$ dependence is peaked near zero and can be dropped from the $\Phi$ fields. In that case, we can transform $\delta \chi^{-1}$ from $\omega^{\prime}$ back to time and obtain a quasistatic approximation for the nematic susceptibility correction $\delta \chi^{-1}(\omega, t)$.

In Fig. 4, we depict numerical computations of $\delta \chi^{-1}\left(\omega, \omega^{\prime}\right)$ for a pulse of the form

$$
\varepsilon(t)=\frac{\hat{x}+\hat{y}}{\sqrt{2}} \varepsilon_{0} \cos (\Omega t) e^{-|t| / \tau_{\text {pulse }},}
$$

where we used the following parameters: $\Gamma_{\mathrm{ph}}=0.05 \Omega_{\mathrm{ph}}$, $\tau_{\text {pulse }}=6 \pi / \Gamma_{\mathrm{ph}}$. To obtain clear-looking images, we tuned the pulse near resonance, $\Omega=\Omega_{\mathrm{ph}}-\Gamma_{\mathrm{ph}} / 2$. Aside from the retarded component, in Fig. 4 we also present the Keldysh 


\section{$\begin{array}{lllll}-1.0 & -0.5 & 0 & 0.5 & 1.0\end{array}$}

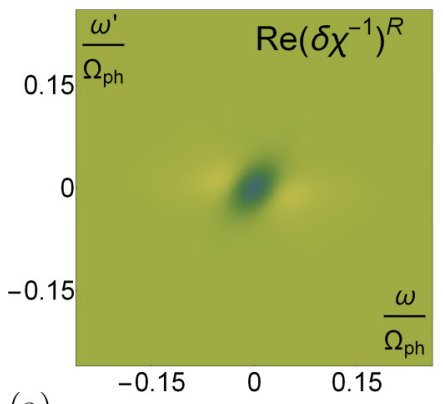

(a)

FIG. 4. The (normalized) retarded (a), (b) and Keldysh (c), (d) components of the correction to the nematic susceptibility $\delta\left(\chi^{-1}\right)^{R / K}$ for a pulse at a frequency near the resonant phonon frequency. For clarity, we divided the Keldysh components by 2 , since in equilibrium $\left|G^{K}\right|=2\left|\operatorname{Im} G^{R}\right|$.

component of $\delta \chi^{-1}$. Figure 4 shows that the quasistatic approximation is indeed a good one for these parameters. Indeed, in Fig. 4 the width of $\delta \chi^{-1}$ is narrower on the $\omega^{\prime}$ axis than on the $\omega$ axis, implying that $\delta \chi^{-1}(\omega, t)$ evolves slowly in time for those frequency scales in $\omega$ where $\delta \chi^{-1}$ is significant.

We verified that the result does not change qualitatively for larger detuning. In Fig. 5, we depict the correction to the nematic susceptibility for an off-resonant pulse with $\Omega=\Omega_{\mathrm{ph}}-7 \Gamma_{\mathrm{ph}} / 2$, and it can be seen that the quasistatic approximation is still a good one. Furthermore, for the offresonant pulse the correction to the nematic susceptibility has a quasiequilibrium form. This can be seen visually in Fig. 5, where the retarded and Keldysh components of $\delta \chi^{-1}$ show an approximate agreement with the equilibrium FDT, see Eq. (29) and the discussion surrounding it. To see this, recall that by construction, $\left(\delta \chi^{-1}\right)^{\mathrm{cl}, \mathrm{cl}}=0$, while from the figure $\left(\delta \chi^{-1}\right)^{K} \approx-2 i \sigma(\omega) \operatorname{Im}\left(\delta \chi^{-1}\right)^{R}$, where $\sigma(x)$ is the sign function. Inverting the matrix yields $\delta \chi^{K} \approx 2 i \sigma(\omega) \operatorname{Im}(\delta \chi)^{R}$. However, for the on-resonant pulse depicted in Fig. 4 , the nematic order-parameter is not in quasiequilibrium, as can be seen by, e.g., the significant induced real part of $\left(\delta \chi^{-1}\right)^{K}$. In both cases, however, there is an induced steady-state nonzero order parameter beyond some critical field strength.

\section{ULTRAFAST CONTROL OF NEMATIC ORDER IN FeSe}

We now apply the results from the previous section to study the evolution of the nematic order parameter in real time in response to a pulse of finite width. We show that it is possible to control the nematic order parameter and to perform a quench of the nematic phase transition.

In Fig. 4, we displayed the field-induced correction $\delta \chi^{-1}\left(\omega, \omega^{\prime}\right)$ to the nematic effective action in response to a pulse in near resonance with $\Omega_{\mathrm{ph}}$. We calculate the classical response to the pulse by solving Eqs. (17)-(19) for the same pulse, with the values of $\lambda=0.04, \varepsilon_{0}=0.04$. Figure 6 (a) depicts the evolution of both the nematic and phononic degrees of freedom in response to the pulse. It can be seen that $\phi(t)$ (blue curve) is initialized with a small nonzero value, and decays until the pulse (black curve) kicks in and enhances the nematic order parameter well above its initial value, but only after the phonon mode has been excited (red curve). In addition, in Fig. 6(b) we present $\left(\delta \chi^{-1}\right)^{R}(\omega, t)$, which is just the inverse Fourier transform of $\left(\delta \chi^{-1}\right)^{R}\left(\omega, \omega^{\prime}\right)$. The real part has a finite contribution at zero frequency for the duration of the pulse, which is equivalent to the shift $r_{\varepsilon}$ of the nematic collective mode in Eq. (21). Similarly, the

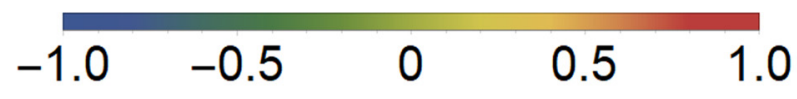

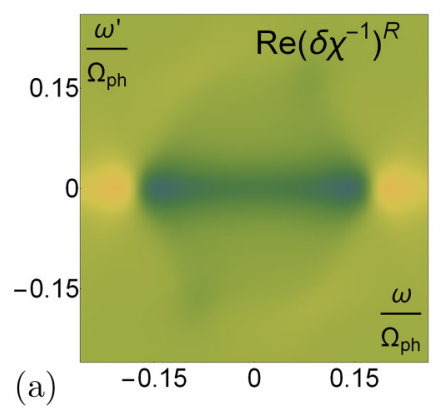

FIG. 5. The (normalized) retarded (a), (b) and Keldysh (c), (d) components of the correction to the nematic susceptibility $\delta\left(\chi^{-1}\right)^{R / K}$ for a pulse at a frequency away from the resonant phonon frequency. For clarity, we divided the Keldysh components by 2 , since in equilibrium $\left|G^{K}\right|=2\left|\operatorname{Im} G^{R}\right|$.
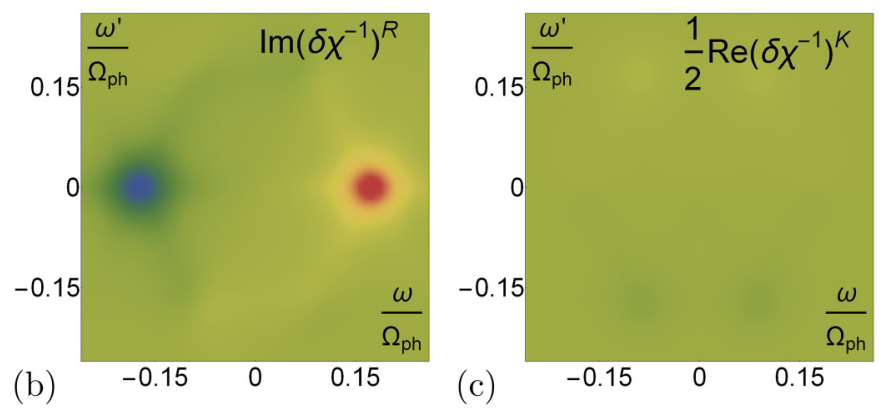

(c)

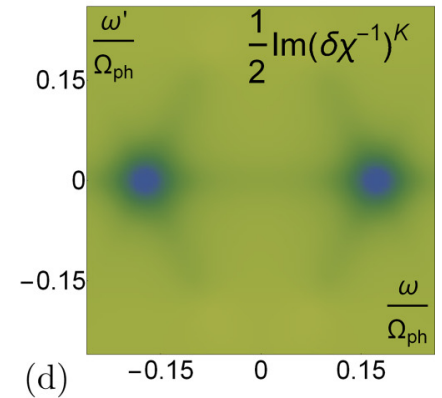

(d) 
(a)

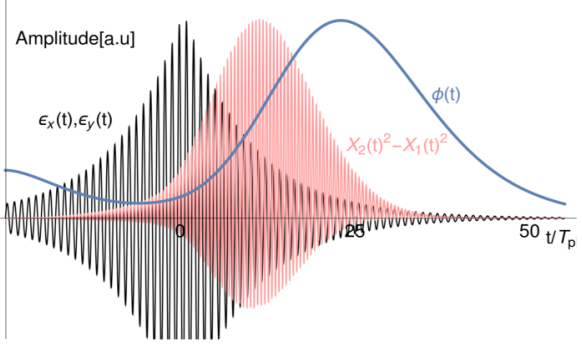

(b)

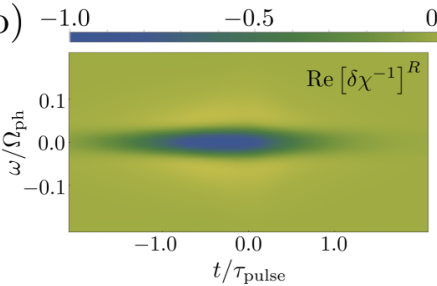

0.0

0.5

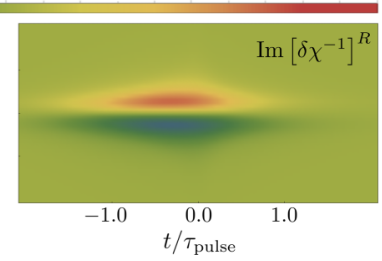

FIG. 6. Evolution of the nematic order parameter in the case of a laser pulse of duration $\tau_{\text {pulse }}$. (a) Classical evolution of $\phi(t)$ and $X_{2}^{2}(t)-X_{1}^{2}(t)$ in response to the same pulse. Note that the phonon modes are excited first by the pulse, and only later the nematic field responds. Here, $T_{\mathrm{ph}}=2 \pi / \Omega_{\mathrm{ph}}$. (b) Real and imaginary parts of the correction to the nematic susceptibility. See Appendix A for details on the parameters used.

imaginary part has an odd-in-frequency contribution whose coefficient is equivalent to $\Gamma_{\varepsilon}$ of the nematic collective mode in Eq. (22).

These results support a feasible protocol for a quantum quench near a putative nematic QCP, consisting of a pulse with frequency $\Omega \sim \Omega_{\mathrm{ph}}$ and length $\tau_{\text {pulse }}$, which excites both components of the $E_{u}$ mode equally. The other relevant timescales for this protocol are the characteristic nematic time $\Omega_{\mathrm{nem}}^{-1} \sim \min \left(r^{-1 / 2}, \Gamma_{\mathrm{nem}} r^{-1}\right)$, the phonon decay time $\Gamma_{\mathrm{ph}}^{-1} \gg$ $\Omega_{\mathrm{ph}}^{-1}$, and $\tau_{\mathrm{el}}$, the electronic heating time. To establish coherent dynamics, the latter must be the longest timescale of the problem, i.e., $\tau_{\mathrm{el}} \gg \Omega_{\mathrm{nem}}^{-1}, \Gamma_{\mathrm{ph}}^{-1}$. We expect this to be the case because, in contrast to traditional pump-probe experiments, our method excites the lattice directly while electronic heating occurs indirectly [41,42].

The excitation of the phonon mode occurs on a timescale $\tau_{\mathrm{ph}} \sim \Gamma_{\mathrm{ph}}^{-1}$ for a resonant laser pulse or $\tau_{\mathrm{ph}} \sim \Omega_{\mathrm{ph}}^{-1}$ for an off-resonant pulse. If $\tau_{\mathrm{ph}} \gg \Omega_{\mathrm{nem}}^{-1}$, as is expected for an onresonance excitation fairly far from the nematic transition, the modification of the nematic behavior is quasistatic and the pole of the nematic susceptibility is dissipative. For $\tau_{\mathrm{ph}} \ll$ $\Omega_{\text {nem }}^{-1}$, as expected near the critical point or for a sufficiently off-resonant laser pulse, the induced softening of the nematic mode [Eq. (21)] is essentially instantaneous. This results in a quantum quench of the nematic instability.

To show that these conditions can be realized in actual systems, we consider the case of FeSe [30,43]. This iron-based superconductor $\left(T_{\mathrm{SC}} \approx 8 \mathrm{~K}\right)$ displays an electronic nematic transition at $T_{\text {nem }} \approx 90 \mathrm{~K}$, that is suppressed to zero upon $\mathrm{S}$ doping, suggesting a putative metallic nematic QCP $[44,45]$. Because $\mathrm{FeSe}_{1-x} \mathrm{~S}_{x}$ does not display long-range magnetic order at ambient pressure, it is an ideal system for studying the interplay of nematicity and superconductivity. Here, we present estimates of the relevant parameters, and the details of how we extracted the numbers appear in Appendix B. We estimate from existing data on $\mathrm{FeSe}$ and related compounds [46-62] that $\hbar \Omega_{\mathrm{ph}} \sim 30 \mathrm{meV}, \hbar \Gamma_{\mathrm{ph}} \sim 0.5-1.5 \mathrm{meV}$, and $\hbar \Omega_{\text {nem }} \sim 10 \mathrm{meV}$ away from the transition, softening further as one approaches it. In addition, we estimate $\tau_{\mathrm{el}}^{-1}<$ $0.25 \mathrm{meV}$ away from the transition, which can get as small as $0.04 \mathrm{meV}$ approaching the transition. These estimates show that the quantum quench regime is achievable in FeSe.

We use these parameters to also estimate the coupling $\lambda$ and, thus, the expected maximum shift in the nematic susceptibility. As shown in Eq. (18), static nematic order $\langle\phi\rangle \neq 0$ splits the frequency of the $E_{u}$ mode by $\hbar \Delta \Omega \approx 2 \lambda\langle\phi\rangle$. We estimate $\langle\phi\rangle$ from the elliptical distortion of the Fermi surface measured by angle-resolved photoemission spectroscopy (ARPES), $\langle\phi\rangle \approx \Delta k_{F} / k_{F}$. This leads to $\lambda / \hbar \Omega_{\mathrm{ph}} \sim 0.04$. The maximum shift of the nematic transition temperature can then be obtained by a Lindemann criterion argument: The maximum possible occupation $n_{\mathrm{ph}}^{L}$ will have $\left\langle X^{2}\right\rangle \approx a^{2} n_{\mathrm{ph}}^{L} \approx c_{L}^{2} \ell^{2}$, where $\ell=3.7 \AA$ is the FeSe lattice constant, and $c_{L}$ is usually [46] a fraction of the order $c_{L}=0.15$. Using these values and a conservative estimate $n_{\mathrm{ph}}=0.15 n_{\mathrm{ph}}^{L}$, we find a shift of $90 \mathrm{~K}$ in the nematic transition temperature (about $4 \mathrm{~K}$ per phonon), indicating the experimental feasibility of a quantum quench both to study and to control the nematic phase over a wide temperature range. Since $T_{S}=90 \mathrm{~K}$ in undoped FeSe, this range is enough to move pristine samples to the vicinity of the QCP at low temperatures, thereby permitting a direct study of the effects of nematic fluctuations on the superconducting $T_{c}$. While the size of this shift may seem surprising, it is a natural result of the fact that energy scales in FeSe, as we showed above, are always on order of several meV, which translates to tens of Kelvin. We caution, however, that at such field strength we also expect some modification of the electronic band structure, due to the indirect coupling of the $E_{u}$ phonon to the electronic density via an $A_{1 g}$ phonon. In addition, if the nematic order parameter is not small, other nonlinear effects, such as magnetism, may appear.

\section{SUMMARY}

In this paper, we presented a theory for the coherent control of a nematic order parameter via laser excitation. We showed that the nonequilibrium excitation of the infrared-active $E_{u}$ phonon mode present in tetragonal systems mediates electronic interactions in the nematic channel.

Besides establishing a robust protocol for light control of nematicity, this result unveils a promising and experimentally feasible avenue to induce a quantum quench across the transition of correlated materials that display nematic order. Because nematic fluctuations are intimately connected to other electronic instabilities, most notably superconductivity and magnetism, the nonequilibrium excitation of nematic fluctuations may also be used to drive transient states with different types of electronic orders. In particular, suppressing nematicity can indirectly enhance superconductivity, since these are known competing orders [35]. 


\section{ACKNOWLEDGMENTS}

We are grateful to A. Chubukov, M. Gastiasoro, A. Kamenev, P. Orth, M. Schütt, and M. Sentef for fruitful discussions, and to Y. Albeck and C. Giannetti for instruction on ultrafast experimental techniques. This work (R.M.F. and M.H.C.) was supported by the U.S. Department of Energy, Office of Science, Basic Energy Sciences, under Award No. DE-SC0012336 and, during completion of the work, under Award No. DE-SC0020045.

\section{APPENDIX A: EVOLUTION OF THE NEMATIC PROPAGATOR'S POLES FOR GENERIC PARAMETERS}

In this Appendix, we will consider other representative solutions to Eq. (17) of the main text that were not discussed in the paper. Specifically, we will relax the conditions that $\sqrt{r}$ is the smallest energy scale in the problem. We solve Eq. (17) of the main text, which we reproduce here for convenience:

$$
\omega^{2}=r-i \Gamma_{\mathrm{nem}} \omega+\frac{\lambda^{2} \chi_{0}}{\hbar \Omega_{\mathrm{ph}}} n_{\mathrm{ph}}\left[d^{R}(\omega+\Omega)+d^{R}(\omega-\Omega)\right] .
$$

We interpret the solution using the classical equations of motion, Eqs. (A2) and (A3), which we again reproduce here:

$$
\begin{gathered}
\ddot{X}_{i}+\Gamma_{\mathrm{ph}} \dot{X}_{i}+\left(\Omega_{\mathrm{ph}}^{2} \pm \frac{2 \lambda}{M a^{2}} \phi\right) X_{i}=\frac{q \varepsilon_{0}}{\sqrt{2} M} \cos \Omega t, \\
\ddot{\phi}+\Gamma_{\mathrm{nem}} \dot{\phi}+r \phi+u \phi^{3}+\frac{\lambda \chi_{0}}{a^{2}}\left(X_{1}^{2}-X_{2}^{2}\right)=0 .
\end{gathered}
$$

In the figures that follow, we present the numerical solution of the pole equation, Eq. (A1) and of the classical equations of motion, Eqs. (A2) and (A3), for several representative situations. We first present the solutions for the disordered phase $r>0$, when the beam pushes the system toward the ordered phase, and then present the solutions for the case $r<0$, when the beam pushes the system out of the ordered phase. For completeness, we also include the case considered in the main text and cite the specific parameter values used to produce the figures in the paper. To reduce the number of parameters in Eqs. (A1)-(A3) we perform the

(a)

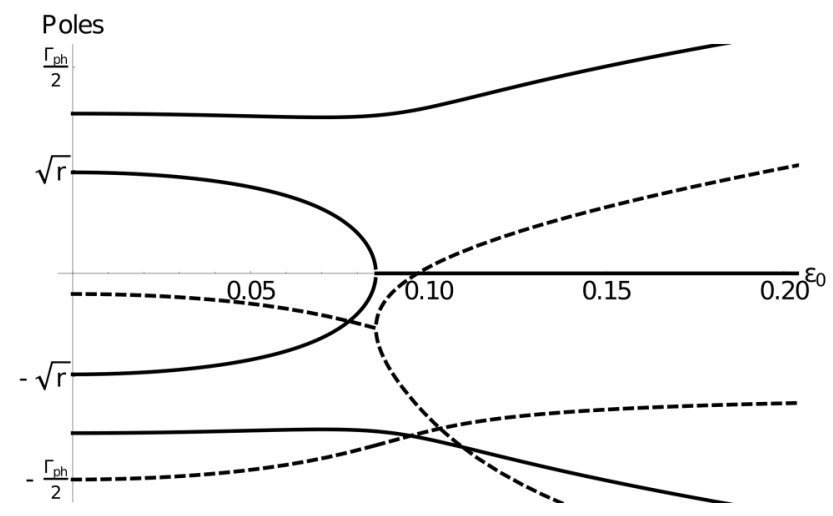

following rescalings: $\lambda \rightarrow \lambda / \hbar, \hbar \chi_{0} \rightarrow \chi_{0}, X_{i} \rightarrow X_{i} / a$, and $q \epsilon_{i} / M a \rightarrow \epsilon_{i}$. In all cases, we measure frequencies in units of $\Omega_{\mathrm{ph}}=1$ and set $\lambda=0.01, \chi_{0}=1$, and $u=1$.

Before continuing, a comment is in order concerning the solution of the classical equation of motion for $\phi$. The classical solution requires a nonzero initial value of the nematic order parameter $\phi(t=0)=\phi(0)$, otherwise there is no time-evolution. In the figures below, we have chosen $\phi(0)=$ 0.031. It is easy to see from the figures, e.g., Fig. 8, that the eventual steady-state configuration of the system is not determined by this initial condition. In an actual experiment, $\phi(0)$ represents the instantaneous local value of the nematic field and not the equilibrium value. A nonzero initial value is a consequence of either fluctuations or an electromagnetic field whose components do not obey $\varepsilon_{1}=\varepsilon_{2}$ exactly [see Eq. (12)].

The specific cases shown here consider various limits with either $\sqrt{r}<\Gamma_{\mathrm{ph}}$ or $\sqrt{r} \gg \Gamma_{\mathrm{ph}}$. They also cover cases where the detuning of the laser, $\left|\Omega_{\mathrm{ph}}-\Omega\right|$, is either comparable to or much larger than the phonon damping, $\Gamma_{\mathrm{ph}}$. In addition, we show solutions when $\Gamma_{\text {nem }}$ is large, of the order of $\Omega_{\mathrm{ph}}$.

\section{Disordered phase $(r>0)$}

Figures $7-10$ are for a red-detuned beam $\left(\Omega<\Omega_{\mathrm{ph}}\right)$, implying that the system is driven toward the nematic phase. Specific parameters for each case are shown in the figure captions; solid (dashed) lines denote the real (imaginary) parts of the poles. Note that, in all cases, there is steady-state nematic order for large enough electric fields, when the imaginary part of one of the poles becomes positive.

\section{Ordered phase $(r<0)$}

As we discussed in the main text, the solutions of Eqs. (A2) and (A3) are more complex in the ordered phase due to the static nonzero value of the order parameter. As we did there, here we again linearize the equations for small $\phi$, to obtain Eqs. (23)-(25) which we reproduce here. The equation for $\phi$ is

$$
\ddot{\phi}+\left(\Gamma_{\mathrm{nem}}+\Gamma_{\varepsilon}\right) \dot{\phi}+\left(r+r_{\varepsilon}\right) \phi+u \phi^{3}=0,
$$

(b)

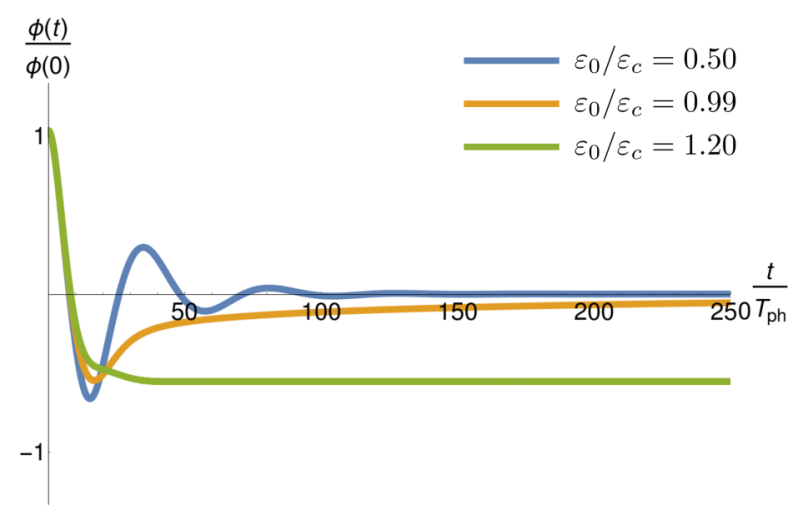

FIG. 7. Evolution of the poles of the nematic propagator (a) and classical evolution of the nematic order parameter (b) for the case presented in the main text. Here $\sqrt{r}=0.025, \Gamma_{\mathrm{ph}}=0.1$, and $\Gamma_{\mathrm{nem}}=0.01$. The laser is red detuned from the phonon resonance and is set at $\Omega=0.96$ (recall $\Omega_{\mathrm{ph}}=1$ ). The critical field is $\varepsilon_{0, c} \approx 0.097$. 
(a)

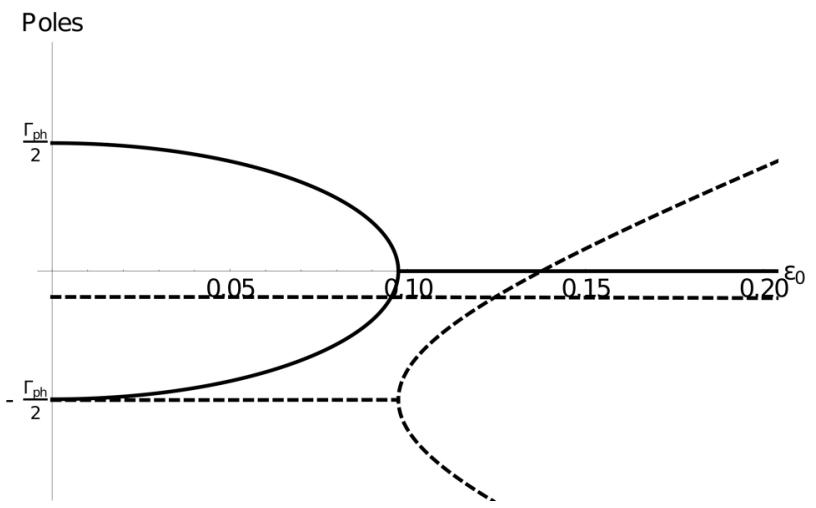

(b)

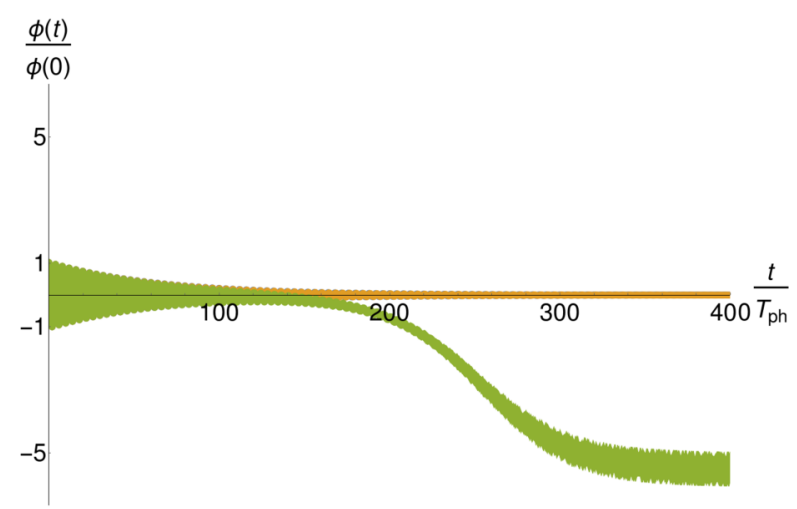

FIG. 8. Evolution of the poles of the nematic propagator (a) and classical evolution of the nematic order parameter (b) when $\sqrt{r} \gg \Gamma_{\mathrm{ph}}$. Specifically, $\sqrt{r}=0.25, \Gamma_{\mathrm{ph}}=0.025, \Gamma_{\mathrm{nem}}=0.005$, and the laser is red detuned, with frequency $\Omega=0.9875$. The critical field is $\varepsilon_{0, c} \approx 0.138$. The color code in the right panel is the same as in Fig. 7; the blue curve is hidden behind the yellow one.

(a)

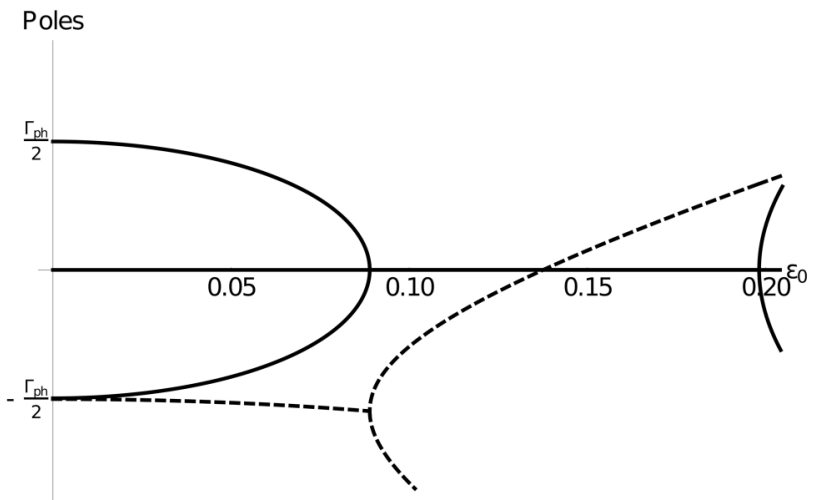

(b)

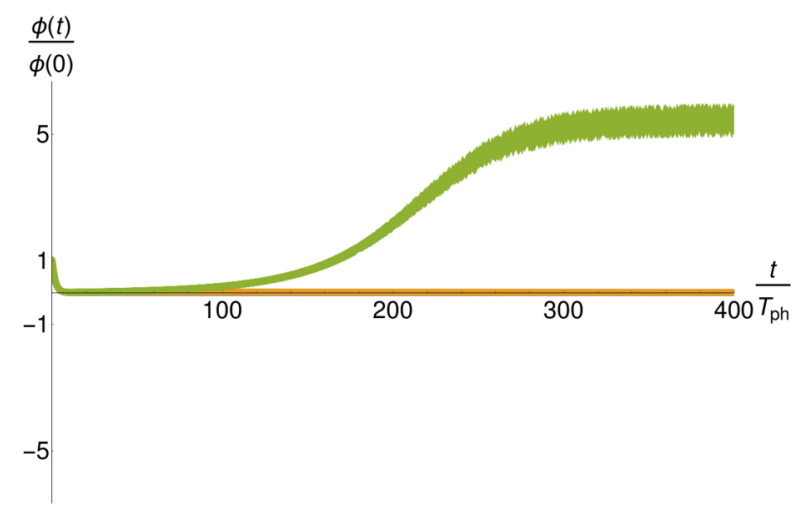

FIG. 9. Evolution of the poles of the nematic propagator (a) and classical evolution of the nematic order parameter (b) for the case where the Landau damping of the nematic mode is comparable to the phonon resonance, $\Omega_{\mathrm{ph}} \sim \Gamma_{\mathrm{nem}}$. Specifically, we choose $\sqrt{r}=0.25, \Gamma_{\mathrm{ph}}=0.025$, $\Gamma_{\text {nem }}=0.8$, and $\Omega=0.9875$. The critical field is $\varepsilon_{0, c} \approx 0.138$ (identical to the case shown in Fig. 8). The color code in the right panel is the same as in Fig. 7; the blue curve is hidden behind the yellow one.

(a)

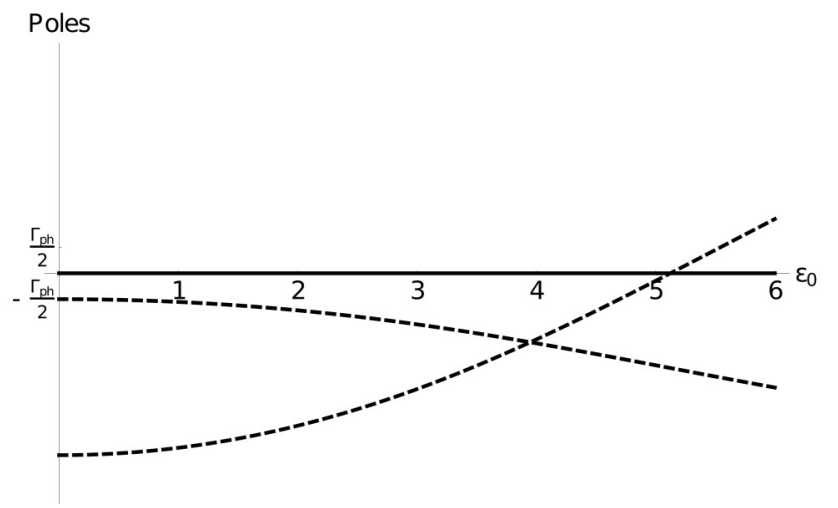

(b)

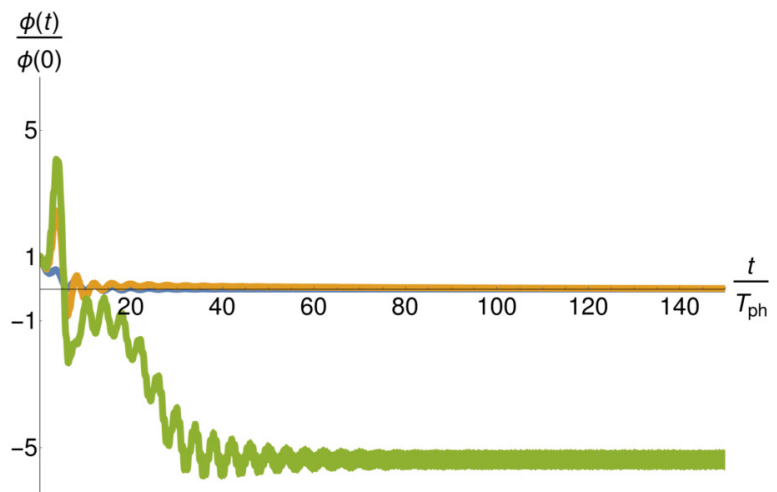

FIG. 10. Evolution of the poles of the nematic propagator (a) and classical evolution of the nematic order parameter (b) for the case where the Landau damping of the nematic mode is comparable to the phonon resonance and the laser detuning is large compared to the phonon damping, $\Omega_{\mathrm{ph}}-\Omega \gg \Gamma_{\mathrm{ph}}$. The values used here are $\sqrt{r}=0.25, \Gamma_{\mathrm{ph}}=0.025, \Gamma_{\mathrm{nem}}=0.8$, and $\Omega=0.75$. The critical field is $\varepsilon_{0, c} \approx 5.13$. The color code in the right panel is the same as in Fig. 7. 


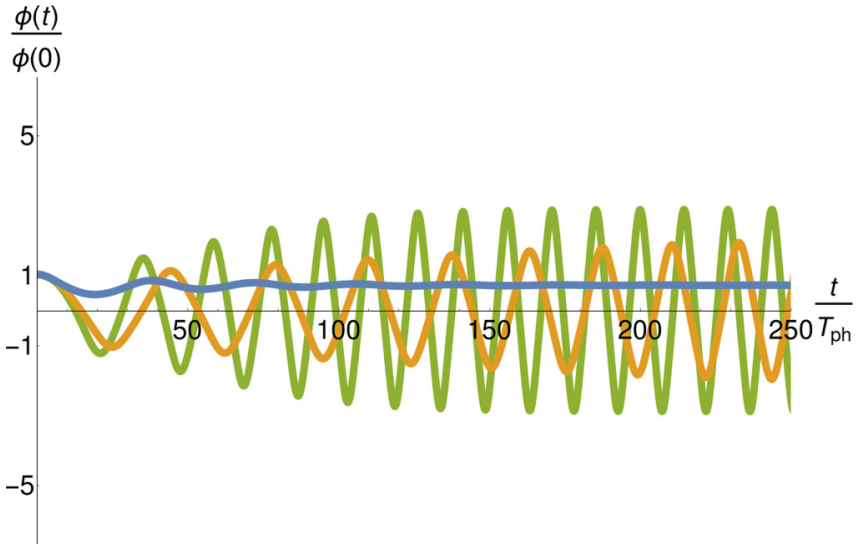

FIG. 11. Classical evolution of the nematic order parameter for the case where $r<0$, and the laser is used to quench the nematic order. This figure is the same as Fig. 3 in the main text. Here $\sqrt{-r}=$ $0.025, \Gamma_{\mathrm{ph}}=0.1$, and $\Gamma_{\text {nem }}=0.01$. The laser is blue detuned from the phonon resonance and is set at $\Omega=1.025$ (recall $\Omega_{\mathrm{ph}}=1$ ). The critical field is $\varepsilon_{0, c} \approx 0.120$. The color code is the same as in Fig. 7.

where $\Gamma_{\varepsilon}, r_{\varepsilon}$ were defined in Eqs. (21) and (22) of the main text. The static order parameter takes the value

$$
\langle\phi\rangle= \pm \sqrt{\frac{-\left(r+r_{\varepsilon}\right)}{u}},
$$

and the dynamics of the $\varphi(t)$, where $\phi(t)=\langle\phi\rangle+\varphi(t)$, are

$$
\ddot{\varphi}+\left(\Gamma_{\text {nem }}+\Gamma_{\varepsilon}\right) \dot{\varphi}-2\left(r+r_{\varepsilon}\right) \varphi=0,
$$

which describes stable oscillations around the order parameter value.

In Figs. 11-13, we present solutions of the equations of motion for several initial conditions with a blue-detuned beam $\left(\Omega>\Omega_{\mathrm{ph}}\right)$. In all cases, the system is driven out of the nematic phase when the electric field strength is larger than a critical

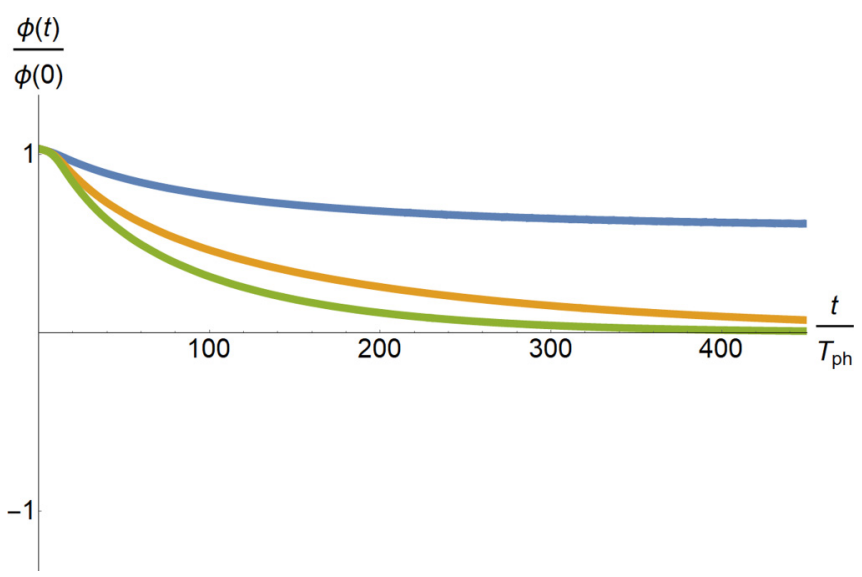

FIG. 12. Classical evolution of the nematic order parameter for the case where $r<0$, and the laser is used to quench the nematic order. In this specific case, $\sqrt{-r} \ll \Gamma_{\mathrm{ph}}$ and the Landau damping of the nematic propagator is assumed comparable to the phonon resonance frequency, $\Gamma_{\text {nem }} \sim \Omega_{\mathrm{ph}}$. To be concrete, we choose $\sqrt{-r}=$ $0.025, \Gamma_{\mathrm{ph}}=0.1$, and $\Gamma_{\mathrm{nem}}=0.8$. The laser is blue detuned from the phonon resonance and is set at $\Omega=1.0125$. The critical field is $\varepsilon_{0, c} \approx 0.145$. The color code is the same as in Fig. 7 .

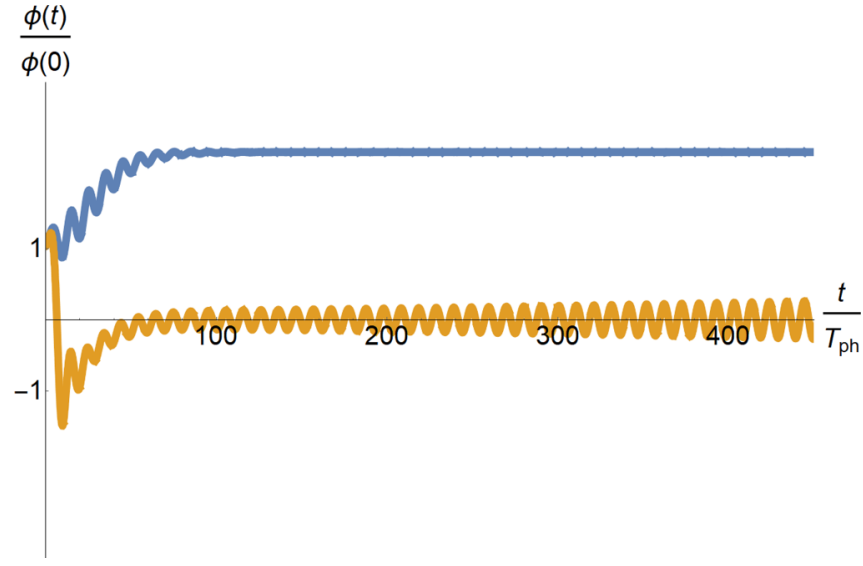

FIG. 13. Classical evolution of the nematic order parameter for the case where $r<0$ and the laser is used to quench the nematic order. In this case, the Landau damping of the nematic propagator is assumed comparable to the phonon resonance frequency, $\Gamma_{\text {nem }} \sim$ $\Omega_{\mathrm{ph}}$, while the detuning, $\Omega-\Omega_{\mathrm{ph}} \gg \Gamma_{\mathrm{ph}}$ and $\sqrt{-r} \gg \Gamma_{\mathrm{ph}}$. Here, $\sqrt{-r}=0.25, \Gamma_{\mathrm{ph}}=0.025$, and $\Gamma_{\text {nem }}=0.8$. The laser is blue detuned from the phonon resonance and is set at $\Omega=1.1$. The critical field is $\varepsilon_{0, c} \approx 0.979$. The color code is the same as in Fig. 7; for clarity, the largest field (green curve) is omitted.

value $\varepsilon_{0, c}$. Specific parameters for each case are displayed in the figure captions. We do not present figures for the pole evolution for these parameter sets, since after the linearization in $\varphi$, all the pole information is already included in Eq. (A6).

\section{Details of the numerical solutions}

The positions of the poles depicted in Fig. 2 of the main text and Figs. 7-10 in the Appendix were obtained from analytical solution of Eq. (A1). The classical trajectories in these figures, in Figs. 11-13, and in Figs. 2 and 6 of the main text were obtained by numerical integration of Eqs. (A2) and (A3). The maps of $\delta \chi^{-1}$ in Fig. 6 were obtained by first numerically integrating the expression in Eq. (30), and convolving the result by a Gaussian mask that suppresses large frequencies, $\left|\omega, \omega^{\prime}\right|>1 / 3$. This was done to remove the highfrequency components of the field. This was then transformed back to the time domain by a numerical Fourier transform. The maps in Figs. 4 and 5 were obtained by numerically integrating the expression in Eq. (30).

\section{APPENDIX B: ESTIMATES OF THE EXPERIMENTAL PARAMETERS FOR FeSe}

In the main text, we invoked the iron-based superconductor $\mathrm{FeSe}_{1-x} \mathrm{~S}_{x}$ to demonstrate the feasibility of our quantum quench protocol. In this section, we briefly outline the sources and methods used to extract experimental parameters for this compound. FeSe has been widely studied, due at least partly to the fact that there are clean single crystals available, and that it does not show long-range magnetic order at ambient pressure [30]. Nevertheless, we were unable to find experimental data for all the parameters used in our theory, and therefore supplemented with data from other, related Fe-based superconductors, most prominently FeTe and $\mathrm{BaFe}_{2} \mathrm{As}_{2}$. 
TABLE I. Estimated experimental parameters for the Fe-based superconductor FeSe. Energies are quoted in $\mathrm{meV}$, and wavevectors in $\AA^{-1}$.

\begin{tabular}{ccccccc}
\hline \hline$\hbar \Omega_{\mathrm{ph}}$ & $\hbar \Gamma_{\mathrm{ph}}$ & $\hbar \Omega_{\mathrm{nem}}$ & $\hbar \tau_{\mathrm{el}}^{-1}$ & $E_{F}$ & $k_{F}$ & $\Delta k_{F}$ \\
\hline $30-33$ & $0.4-1.5$ & 10 & 0.24 & 25 & 0.13 & 0.02 \\
\hline \hline
\end{tabular}

Most of the external parameters in our theory are directly measurable quantities. The exceptions are the coupling $\lambda$, the maximum nematic order parameter strength $\langle\phi\rangle$, and the bare nematic susceptibility $\chi_{0} / r$. In general, the last two can only be extracted from experiment up to a prefactor (which can also be temperature dependent). In a Stoner-type theory for the nematic transition, $\chi_{0} / r_{0} \sim N_{F}$, where $r_{0}$ is the bare nematic gap (at high temperatures) and $N_{F}$ is the density of states at the Fermi level. Since the nematic susceptibility diverges at the nematic transition temperature $T_{\text {nem }}$ with the meanfield behavior $\left(T-T_{\text {nem }}\right)^{-1}$, we estimate in these systems that $\chi_{0}^{-1} r=E_{F}\left(T-T_{\text {nem }}\right) / T_{\text {nem. }}$. As mentioned in the main text, we took $\Delta k_{F} / k_{F}$ as a proxy for $\langle\phi\rangle$, which is again only correct up to an unknown prefactor. We stress that the uncertainty in our knowledge of $\chi_{0}^{-1} r$ and $\langle\phi\rangle$ means that our estimates for the effective coupling are only valid to within an order of magnitude.

For clarity, we have compiled our estimates of the various parameters for FeSe into Table I. We now briefly outline what sources we used to extract the experimental parameters in Table I and our estimate for the coupling, which, as noted in the paper, is

$$
\lambda=\frac{\Delta \Omega}{2\langle\phi\rangle},
$$

where $\Delta \Omega$ is the splitting of the $E_{u}$ mode in the nematic phase.

The infrared phonon structure and dispersion relations in FeSe have been both calculated [49-51] and detected experimentally [47,52-54]. We discuss data only for the tetragonal phase, above $T_{\mathrm{nem}} \simeq 90 \mathrm{~K}$. Reference [52] reported $\hbar \Omega_{\mathrm{ph}}=$ $30.9 \mathrm{meV}$ in a film of $\mathrm{FeSe}$ on $\mathrm{CaF}_{2}$, measured by optical reflectometry. Reference [47] reported $\hbar \Omega_{\mathrm{ph}}=32 \mathrm{meV}$ near the $\bar{M}$ point, measured by electron energy-loss spectroscopy on a single crystal. Reference [54] reported $\hbar \Omega_{\mathrm{ph}}=31.3 \mathrm{meV}$ in neutron scattering. This tallies with theoretical calculations [49,51] predicting $\hbar \Omega_{\mathrm{ph}}=30-35 \mathrm{meV}$, and only a weak dispersion for the $E_{u}$ mode. We did not find a reported measurement of $\Gamma_{\mathrm{ph}}$ for FeSe. However, Ref. [55] reported $\hbar \Gamma_{\mathrm{ph}} \sim 1.2-1.5 \mathrm{meV}$ in the related chalcogenides FeTe and $\mathrm{FeTe}_{1-x} \mathrm{Se}_{x}$. For FeSe $B_{1 g}$ optical mode, Ref. [56] reported a decay rate $\hbar \Gamma_{B_{18}} \approx 0.4 \mathrm{meV}$ at the nematic transition.

The electronic structure and dynamics of FeSe have been extensively studied by (among others) ARPES and Raman techniques. To estimate the relevant timescale $\Omega_{\text {nem }}^{-1}$, we considered reports of polarization-resolved Raman data measuring the dynamic response of the nematic mode in $\mathrm{FeSe}_{1-x} \mathrm{~S}_{x}$ $[57,58]$. These measurements show a wide damped peak centered around $25 \mathrm{meV}$, and extending to about $50 \mathrm{meV}$ before beginning to decay. Although there are interesting features in the entire region (for details see, e.g., Ref. [58]), the sharpest features show up at frequencies below about $\hbar \Omega_{\text {nem }} \sim 10-12 \mathrm{meV}$, and soften as one approaches the nematic transition, giving us the estimate for $\Omega_{\text {nem }}$ in Table I. To estimate the coupling constant $\lambda$, we used Eq. (B1). As a proxy for $\langle\phi\rangle$, we took the elliptical distortion of the holelike Fermi surface $\Delta k_{F}=k_{F, x}-k_{F, y}$ at the $Z$ point. We extracted the values for $E_{F}=25 \mathrm{meV}, k_{F}=0.13 \AA^{-1}$ and $\Delta k_{F}=0.02 \AA^{-1}$ from Ref. [43] (all at the $Z$ point). We have not found a detailed study of the $E_{u}$ mode splitting in FeSe. However, the $E_{g}$ mode, which is Raman active but has almost the same resonance frequency as $E_{u}$, has been measured. Reference [59] reports a maximum split of $\hbar \Delta \Omega=0.4 \mathrm{meV}$ at $20 \mathrm{~K}$. These estimates yield $\lambda \approx 1.3 \mathrm{meV}$.

Using these numbers, we obtain an estimate for the shift of the nematic susceptibility. From Eq. (21) of the main text, the maximum shift occurs for

$$
\Omega^{2}=\Omega_{\mathrm{ph}}^{2}(1-\gamma),
$$

where $\gamma=\Gamma_{\mathrm{ph}} / \Omega_{\mathrm{ph}}$. The shift in the nematic transition temperature $T_{\text {nem }}$ is proportional to the shift in $r$. For small $\gamma$, the shift is

$$
\frac{\delta T_{\mathrm{nem}}}{T_{\mathrm{nem}}}=\frac{-r_{\varepsilon}}{\chi_{0} E_{F}} \approx \frac{2 \lambda^{2}}{E_{F} \hbar \Omega_{\mathrm{ph}}} n_{\mathrm{ph}} \times \frac{\Omega_{\mathrm{ph}}}{2 \Gamma_{\mathrm{ph}}} \approx 0.045 n_{\mathrm{ph}} .
$$

Since $T_{\text {nem }} \approx 90 \mathrm{~K}$, this corresponds to about $4 \mathrm{~K}$ per phonon. To estimate the maximum possible phonon occupation number, it is simplest to consider what occupation number would melt the lattice. This can be found from the Lindemann criterion,

$$
n_{\mathrm{ph}}^{L} a^{2}=c_{L}^{2} \ell^{2},
$$

where $\ell=3.7 \AA$ is the $a$-axis lattice constant of FeSe and $c_{L}$ is some fraction (we choose the commonly used value $c_{L}=$ 0.15 [46]) and $a$ is the classical oscillator length, $\sqrt{\hbar / M \Omega_{\mathrm{ph}}}$. Since the $E_{u}$ mode involves motion of both the $\mathrm{Fe}$ and $\mathrm{Se}$ atoms, we use the mass, $M=\sqrt{M_{\mathrm{Fe}} M_{\mathrm{Se}}} \approx 67 u$. Then we find $a=0.045 \AA$, which in turn implies $n_{\mathrm{ph}}^{L}=152$. A conservative estimate for $n_{\mathrm{ph}}$ is $n_{\mathrm{ph}}=0.1-0.2 n_{\mathrm{ph}}^{L}$. To be concrete, we take $n_{\mathrm{ph}}=0.15 n_{\mathrm{ph}}^{L}$ resulting in $\delta T_{\text {nem }} \approx 90 \mathrm{~K}$.

To estimate the equilibration time $\tau_{\mathrm{el}}$, we summed up the phonon decay time $\Gamma_{\mathrm{ph}}^{-1}$ with measured electronic decay times $\Gamma_{\mathrm{el}-\mathrm{ph}}^{-1}$ from ultrafast optical reflectivity experiments. Typically, such measurements heat up the electronic subsystem, which then decays slowly into the lattice $[60,61]$. This decay is characterized by two distinct timescales: a fast decay of the electrons into symmetry-preferred optical phonon modes (e.g., $A_{1 g}$ ) and then a slow anharmoic decay of these modes to the lattice. We took as our estimate for the decay time $\Gamma_{\mathrm{el}-\mathrm{ph}}^{-1}$ the decay constant of this slow anharmonic decay. We also considered temperatures not too close to the critical temperature $T_{\text {nem }}$, out of the assumption that such a timescale roughly characterizes a generic electron-phonon decay. We obtained $\Gamma_{\mathrm{el}-\mathrm{ph}}^{-1}=0.5-0.6 \mathrm{meV}$ from Ref. [60]. We took as our estimate for $\Gamma_{\mathrm{ph}}$ the lower value quoted above in Table I (which is one that was measured for actual FeSe), implying a total decay $\tau_{\mathrm{el}}=\Gamma_{\mathrm{ph}}^{-1}+\Gamma_{\mathrm{el}-\mathrm{ph}}^{-1}=18.8-17.2 \mathrm{ps}$. However, this estimate does not take into account the expected slowing down of electronic heating rates near the nematic transition. Such slowing down has been measured in $\mathrm{BaFe}_{2} \mathrm{As}_{2}$ [62]. 
[1] A. Mitra, Annu. Rev. Condens. Matter Phys. 9, 245 (2016).

[2] A. F. Kemper, M. A. Sentef, B. Moritz, T. P. Devereaux, and J. K. Freericks, Ann. Phys. 529, 1600235 (2017).

[3] M. Heyl, Rep. Prog. Phys. 81, 054001 (2018).

[4] J. Orenstein, Phys. Today 65(9), 44 (2012).

[5] A. von Hoegen, R. Mankowsky, M. Fechner, M. Först, and A. Cavalleri, Nature 555, 79 (2018).

[6] C. Giannetti, M. Capone, D. Fausti, M. Fabrizio, F. Parmigiani, and D. Mihailovic, Adv. Phys. 65, 58 (2016).

[7] M. Mitrano, A. Cantaluppi, D. Nicoletti, S. Kaiser, A. Perucchi, S. Lupi, P. Di Pietro, D. Pontiroli, M. Riccò, S. R. Clark, D. Jaksch, and A. Cavalleri, Nature 530, 461 (2016).

[8] M. Knap, M. Babadi, G. Refael, I. Martin, and E. Demler, Phys. Rev. B 94, 214504 (2016).

[9] M. Claassen, D. M. Kennes, M. Zingl, M. A. Sentef, and A. Rubio, Nat. Phys. 15, 766 (2019).

[10] M. Rini, R. Tobey, N. Dean, J. Itatani, Y. Tomioka, Y. Tokura, R. W. Schoenlein, and A. Cavalleri, Nature 449, 72 (2007).

[11] T. F. Nova, A. Cartella, A. Cantaluppi, M. Först, D. Bossini, R. V. Mikhaylovskiy, A. V. Kimel, R. Merlin, and A. Cavalleri, Nat. Phys. 13, 132 (2016).

[12] M. Gandolfi, G. L. Celardo, F. Borgonovi, G. Ferrini, A. Avella, F. Banfi, and C. Giannetti, Phys. Scr. 92, 034004 (2017).

[13] M. Puviani and M. A. Sentef, Phys. Rev. B 98, 165138 (2018).

[14] H. A. Hafez, X. Chai, A. Ibrahim, S. Mondal, D. Férachou, X. Ropagnol, and T. Ozaki, J. Opt. 18, 093004 (2016).

[15] J. A. Fülöp, L. Pálfalvi, and J. Hebling, 2017 XXXIInd General Assembly and Scientific Symposium of the International Union of Radio Science (URSI GASS) (IEEE, Montreal, QC, Canada, 2017), p. 1

[16] M. C. Hoffmann and J. A. Fülöp, J. Phys. D 44, 083001 (2011).

[17] D. Nicoletti and A. Cavalleri, Adv. Opt. Photon. 8, 401 (2016).

[18] G. Chiriacò, A. J. Millis, and I. L. Aleiner, Phys. Rev. B 98, 220510(R) (2018).

[19] A. Subedi, A. Cavalleri, and A. Georges, Phys. Rev. B 89, 220301(R) (2014).

[20] D. M. Kennes, E. Y. Wilner, D. R. Reichman, and A. J. Millis, Nat. Phys. 13, 479 (2017).

[21] Y. Murakami, N. Tsuji, M. Eckstein, and P. Werner, Phys. Rev. B 96, 045125 (2017).

[22] R. M. Fernandes, L. H. VanBebber, S. Bhattacharya, P. Chandra, V. Keppens, D. Mandrus, M. A. McGuire, B. C. Sales, A. S. Sefat, and J. Schmalian, Phys. Rev. Lett. 105, 157003 (2010).

[23] U. Karahasanovic and J. Schmalian, Phys. Rev. B 93, 064520 (2016).

[24] I. Paul and M. Garst, Phys. Rev. Lett. 118, 227601 (2017).

[25] S. A. Kivelson, E. Fradkin, and V. J. Emery, Nature 393, 550 (1998).

[26] E. Fradkin, S. A. Kivelson, M. J. Lawler, J. P. Eisenstein, and A. P. Mackenzie, Annu. Rev. Condens. Matter Phys. 1, 153 (2010).

[27] M. Vojta, Adv. Phys. 58, 699 (2009).

[28] F. Ronning, T. Helm, K. R. Shirer, M. D. Bachmann, L. Balicas, M. K. Chan, B. J. Ramshaw, R. D. McDonald, F. F. Balakirev, M. Jaime, E. D. Bauer, and P. J. W. Moll, Nature 548, 313 (2017).

[29] R. M. Fernandes, A. V. Chubukov, and J. Schmalian, Nat. Phys. 10, 97 (2014).
[30] A. E. Böhmer and A. Kreisel, J. Phys.: Condens. Matter 30, 023001 (2018).

[31] S. Lederer, Y. Schattner, E. Berg, and S. A. Kivelson, Proc. Natl. Acad. Sci. 114, 4905 (2017).

[32] H.-H. Kuo, J.-H. Chu, J. C. Palmstrom, S. A. Kivelson, and I. R. Fisher, Science 352, 958 (2016).

[33] S. Licciardello, J. Buhot, J. Lu, J. Ayres, S. Kasahara, Y. Matsuda, T. Shibauchi, and N. E. Hussey, Nature 567, 213 (2019).

[34] T. A. Miller, R. W. Chhajlany, L. Tagliacozzo, B. Green, S. Kovalev, D. Prabhakaran, M. Lewenstein, M. Gensch, and S. Wall, Nat. Commun. 6, 8175 (2015).

[35] S. Nandi, M. G. Kim, A. Kreyssig, R. M. Fernandes, D. K. Pratt, A. Thaler, N. Ni, S. L. Bud'ko, P. C. Canfield, J. Schmalian, R. J. McQueeney, and A. I. Goldman, Phys. Rev. Lett. 104, 057006 (2010).

[36] M. Schütt, P. P. Orth, A. Levchenko, and R. M. Fernandes, Phys. Rev. B 97, 035135 (2018).

[37] M. A. Sentef, Phys. Rev. B 95, 205111 (2017).

[38] S. Onari and H. Kontani, Phys. Rev. Lett. 109, 137001 (2012)

[39] A. Kamenev, Field Theory of Nonequilibrium Systems (Cambridge University Press, Cambridge, UK, 2011).

[40] M. H. Devoret, A. Kamal, and B. Abdo, in Fluctuating Nonlinear Oscillators, edited by Mark Dykman (Oxford University Press, Oxford, UK, 2012).

[41] P. B. Allen, Phys. Rev. Lett. 59, 1460 (1987)

[42] In principal, the electric field also couples directly to electrons, leading to finite dissipation and heating proportional to the single-electron scattering rate. Measurements of optical conductivity in FeSe [52] indicate that the scattering rate associated with those electronic degrees of freedom that couple to nematicity is $\gamma \sim 14 \mathrm{meV} \ll \Omega_{\mathrm{ph}}$ at $T=100 \mathrm{~K}$, so this heating channel may be neglected for a sufficiently narrow laser.

[43] A. I. Coldea and M. D. Watson, Annu. Rev. Condens. Matter Phys. 9, 125 (2017).

[44] P. Reiss, M. D. Watson, T. K. Kim, A. A. Haghighirad, D. N. Woodruff, M. Bruma, S. J. Clarke, and A. I. Coldea, Phys. Rev. B 96, 121103(R) (2017).

[45] S. Hosoi, K. Matsuura, K. Ishida, H. Wang, Y. Mizukami, T. Watashige, S. Kasahara, Y. Matsuda, and T. Shibauchi, Proc. Natl. Acad. Sci. 113, 8139 (2016).

[46] D. R. Nelson, Defects and Geometry in Condensed Matter Physics (Cambridge University Press, Cambridge, New York, 2002), p. 350 .

[47] K. Zakeri, T. Engelhardt, T. Wolf, and M. Le Tacon, Phys. Rev. B 96, 094531 (2017).

[48] A. Fedorov, A. Yaresko, T. K. Kim, Y. Kushnirenko, E. Haubold, T. Wolf, M. Hoesch, A. Grüneis, B. Büchner, and S. V. Borisenko, Sci. Rep. 6, 36834 (2016).

[49] A. Subedi, L. Zhang, D. J. Singh, and M. H. Du, Phys. Rev. B 78, 134514 (2008)

[50] W. Wang, J. Sun, and S. Li, Appl. Phys. Lett. 99, 082504 (2011).

[51] W. Wang, J. Sun, S. Li, and H. Lu, Physica C: Supercond. 472, 29 (2012)

[52] M. Nakajima, K. Yanase, F. Nabeshima, Y. Imai, A. Maeda, and S. Tajima, Phys. Rev. B 95, 184502 (2017). 
[53] D. Phelan, J. N. Millican, E. L. Thomas, J. B. Leao, Y. Qiu, and R. Paul, Phys. Rev. B 79, 014519 (2009).

[54] V. Ksenofontov, G. Wortmann, A. I. Chumakov, T. Gasi, S. Medvedev, T. M. McQueen, R. J. Cava, and C. Felser, Phys. Rev. B 81, 184510 (2010).

[55] C. C. Homes, Y. M. Dai, J. Schneeloch, R. D. Zhong, and G. D. Gu, Phys. Rev. B 93, 125135 (2016).

[56] V. Gnezdilov, Y. G. Pashkevich, P. Lemmens, D. Wulferding, T. Shevtsova, A. Gusev, D. Chareev, and A. Vasiliev, Phys. Rev. B 87, 144508 (2013).

[57] P. Massat, D. Farina, I. Paul, S. Karlsson, P. Strobel, P. Toulemonde, M.-A. Méasson, M. Cazayous, A. Sacuto, S. Kasahara, T. Shibauchi, Y. Matsuda, and Y. Gallais, Proc. Natl. Acad. Sci. 113, 9177 (2016).

[58] W.-L. Zhang, S.-F. Wu, S. Kasahara, T. Shibauchi, Y. Matsuda, and G. Blumberg, arXiv:1710.09892.
[59] Y. Hu, X. Ren, R. Zhang, H. Luo, S. Kasahara, T. Watashige, T. Shibauchi, P. Dai, Y. Zhang, Y. Matsuda, and Y. Li, Phys. Rev. B 93, 060504(R) (2016).

[60] C. W. Luo, I. H. Wu, P. C. Cheng, J.-Y. Lin, K. H. Wu, T. M. Uen, J. Y. Juang, T. Kobayashi, D. A. Chareev, O. S. Volkova, and A. N. Vasiliev, Phys. Rev. Lett. 108, 257006 (2012).

[61] C. W. Luo, I. H. Wu, P. C. Cheng, J.-Y. Lin, K. H. Wu, T. M. Uen, J. Y. Juang, T. Kobayashi, Y. C. Wen, and T. W. Huang, New J. Phys. 14, 103053 (2012).

[62] A. Patz, T. Li, S. Ran, R. M. Fernandes, J. Schmalian, S. L. Bud'ko, P. C. Canfield, I. E. Perakis, and J. Wang, Nat. Commun. 5, 3229 (2014).

Correction: An award number was missing in the Acknowledgments section and has been inserted. 